\title{
IMPACT OF BAD COMPONENTS ON COSTS AND PRODUCTIVITY IN AUTOMATIC ASSEMBLY
}

\author{
Mathieu Oulevey, Sandra Koelemeijer, Jacques Jacot \\ Laboratoire de Production Microtechnique (LPM) \\ Ecole Polytechnique Fédérale de Lausanne (EPFL) \\ CH-1015 Lausanne, Switzerland \\ mathieu.oulevey@epf.ch
}

\begin{abstract}
This article shows the consequences of using parts with scrap in an automatic assembly system. The scrap is the percentage of parts that do not reach the required conformity and will cause either a defective assembly or a jam in the system. The two consequences considered here are extra cost and loss of productivity. To show this, we will focus on one part assembly operation anywhere in the process, which consists of feeding, assembling the part and inspecting the resulting sub-assembly.

We will show that the effect of scrap can be huge and depends strongly on how the bad part is detected. 4 cases are considered: 1.The parts are sorted before feeding 2.The bad parts jam in the feeder 3.The bad parts jam while assembling 4.The sub-assembly with bad parts is ejected after inspection. By evaluating each case in 3 examples, we will show that case 3 is often the worst. We will also define the conditions where it is better to sort the parts first (1), let them jam in the feeder (2) or sort the sub-assemblies (4).
\end{abstract}

Keywords: Micro-assembly, cost-modeling

\section{INTRODUCTION}

When designing or choosing an assembly installation, it is very important to have a good cost estimation, therefore predictive cost models are of very high interest. A good understanding of where the major production costs lie is essential for a good cost model.

In macro-assembly, a productivity rate (cycle time and setup times) is often enough to describe the line throughput and evaluate the costs () . As products become smaller (high precision and micro-systems assembly), processes are more complex to control, often resulting in unexpected prohibitive assembly costs. Not only assembly processes are more difficult, but also the manufacturing processes of the components, whose yield become very low (Hong et al., 
2005). Scrap is then major source of costs, and is not taken into account in primary cost estimations.

In this paper, we show that the way of specifying components and their tolerances is vital not only for the product's functionality but also for the productivity rate of the assembly installation. We furthermore show that the way to deal with yields and scrap is essential for the good functioning of an assembly installation. Those elements are decisive for the cost of the final product, and this model should be useful to specify the scrap when ordering parts and evaluate the consequences on the product cost. We take the example of defaults in screws on an automatic screw installation to illustrate this.

\section{MODEL DESCRIPTION}

General cost model of product assembly. Assembly costs depend on the cost of ownership of the the installation. This cost is divided into investment cost and running costs. To evaluate the investment costs, we have shown (Oulevey et al., 2003) that the cost of an assembly installation mainly depends on the production cycle time, the components characteristics and the type of processes needed to assemble them. From tables of available processes and feeding systems, we computed the installation cost by estimating a difficulty factor for each component and process. We have also evaluated the running costs, including the building rental, production operators, electricity,... With these elements, we can evaluate the assembly cost of one product as long as the production rate corresponds to the technical cycle-time. If the installation is jammed due to bad parts or produces bad assemblies, the cost of ownership per part and the yield of We will show that scrap has a wide impact on running costs.

Consequences of bad parts on costs. The main causes of non-quality in assembly are components that do not meet specifications (tolerances, functionality,...) and assembly processes that are not perfect (which can generate bad assemblies even it the components were good). The main consequences of this non-quality are

- Cost of bad components

- Cost of good components used in a bad assembly

- Loss of time due to bad assemblies

- Loss of time due to jams in feeders and assembly processes

- Operator cost to unjam

Here, we will focus only on non-quality due to bad parts and assume that the processes will always make good assemblies from good parts. 
Methodology and parameters used to estimate costs. Let us consider an assembly system for a product made of $n$ different parts. The assembly system will be composed of $n$ feeding systems, $n$ assembly processes and usually $n$ quality inspections. Here, we will compute the cost due to the scrap of one part on one process. If more than one part has a scrap, we can use this model recursively. If we focus on one of these $n$ assembly process, we will have 2 inputs (a sub-assembly and a part) and one output (a sub-assembly).

We will compute the assembly extra-cost of one part on a receptor using the parameters in Table 1. In order to have good comparison, we assume that:

- The receptors (sub-assemblies on which the part is assembled) have no defaults.

- The assembly process is zero-default.

- All bad parts will be detected or cause a problem in the same way (see the cases below). If this is not true, the cases can be superposed.

\begin{tabular}{|c|c|}
\hline$\overline{T_{c}}$ & $\begin{array}{l}\text { Cycle time [s]. Mean time to assemble one sub-assembly when the machine is } \\
\text { on. Excludes all down time (jams, setup, } . . \text { ). } \\
\hookrightarrow \text { The hourly productivity is given by } 3600 / T_{c} \text { [parts/h]. }\end{array}$ \\
\hline$T_{j f}$ & $\begin{array}{l}\text { Jam duration in feeder [s]. Mean down time due to a part jam in the feeding } \\
\text { system. }\end{array}$ \\
\hline$T_{j p}$ & $\begin{array}{l}\text { Jam duration in assembly [s]. Mean down time due to a part jam in the as- } \\
\text { sembly system. }\end{array}$ \\
\hline$k$ & Bad parts. Proportion of bad parts. \\
\hline$V_{p}$ & Part value [€]. Value of one part (including the bad ones). \\
\hline$V_{s}$ & $\begin{array}{l}\text { Sub-assembly value }[€] \text {. Value of the sub-system on which the part will be } \\
\text { assembled. If a rework after a bad assembly is cheaper, use this value instead. }\end{array}$ \\
\hline$C_{l i n}$ & $\begin{array}{l}\text { Assembly line cost }[€ / \text { s]. Includes running costs (operators, air and electricity } \\
\text { supplies, ...). and costs of ownership (depreciation or rent, used area, ...). } \\
\hookrightarrow \text { The hourly cost is given by } 3600 \cdot C_{\text {line }}[€ / \mathrm{h}] \text {. }\end{array}$ \\
\hline$C_{o p}$ & $\begin{array}{l}\text { Operator cost }[€ / \mathrm{s}] \text {. Cost of the operator who will deal with jams due to } \\
\text { defective parts. } \\
\hookrightarrow \text { The hourly cost is given by } 3600 \cdot C_{o p}[€ / \mathrm{h}] \text {. }\end{array}$ \\
\hline
\end{tabular}

Table 1. Model parameters

\section{CASES DESCRIPTION}

The extra cost due to bad parts will widely depend on where they are detected. 5 cases are considered:

0 The bad parts have no effect on assembly, as if they were good;

1 The bad parts are removed before feeding (sorting by an operator);

2 The bad parts jam in the feeder; 
3 The bad parts jam on the assembly process;

4 The bad parts cause no jam, but the assembly is detected as bad and ejected during further inspection.

Cases 0 and 1 are trivial but useful for comparison purposes, whereas cases 2 to 4 correspond to the situations we want to analyze. Each case will be explained in the next sections.

Case 0 : No scrap. Let us consider the case where there is no scrap in parts and all sub-assembly are good. We suppose that no other factor will disrupt the production, in order to use the results as a reference for comparison purposes. The production costs are trivial: the hourly productivity $P_{0}$ of the line is directly given by the cycle time, since the machine never stops:

$$
P_{0}=3600 / T_{c}
$$

and the assembly $\operatorname{cost} C_{0}$ is given by the machine cost during one cycle:

$$
C_{0}=C_{\text {line }} / P_{0}
$$

No operators are required in this case.

Case 1 : Sort the parts first. If we decide to sort the parts before feeding them, we have to consider an operating time $T_{i}$ for inspection. This case is not pertinent, because nobody would affect operators to sort the bad parts, but it could give an idea of the part extra cost to have zero-default:

$C_{1}=\frac{C_{\text {line }}}{P_{0}}+\frac{1}{1-k} \cdot T_{i} \cdot C_{o p}$

and the hourly productivity does not change since only good parts are fed:

$$
P_{1}=3600 / T_{c}
$$

Case 2 : Bad parts jam in feeder. If the bad parts jam in the feeder, usually an alarm occurs $T_{a}$ seconds before the production line is stopped. If the operator is close and efficient (i.e. the time to arrive and fix the jam $T_{j}$ is smaller than $T_{a}$ ), the jam will have no incidence on the production. If the operator is far or the jam is difficult to fix $\left(T_{j}>T_{a}\right)$, the machine will be stopped for a time $T_{j}-T_{a}$. Since the average down time requires the probability distributions of $T_{j}$ and $T_{a}$ to be computed, we will use

$$
\begin{aligned}
& P_{2}=3600 /\left(T_{c}+k \cdot T_{j f}\right) \\
& C_{2}=C_{\text {line }}\left[T_{c}+k \cdot T_{j f}\right]+C_{o p}\left[k \cdot T_{j f}\right]
\end{aligned}
$$

Case 3 : Bad parts jam in assembly process. This case is similar to case 2 but worst: firstly because the receptor is damaged and is either trashed or reworked (which costs $V_{s}$ ), secondly because the jam duration $T_{j p}$ is usually greater than $T_{j f}$ in case 2 . The productivity rate is similar to case 2

$$
P_{3}=3600 /\left(T_{c}+k \cdot T_{j p}\right)
$$

but the cost takes into account the damaged receptor:

$$
C_{3}=C_{l i n e}\left[T_{c}+k \cdot T_{j p}\right]+C_{o p}\left[k \cdot T_{j p}\right]+k \cdot V_{s}
$$


Case 4 : Bad parts are detected after assembly (inspection). The subassembly is damaged like in case 3 , but it is ejected without jamming. In this case, The productivity is better, as no jam occur:

$$
P_{4}=3600 /\left(\frac{1}{1-k} T_{c}\right)
$$

and the cost is similar to case 3 , but without time loss due to jams (no operator required):

$$
C_{4}=C_{\text {line }}\left[\frac{1}{1-k} T_{c}\right]+k \cdot V_{s}
$$

\section{EXAMPLES}

Example 1 : single screw. A typical example of extra-costs due to bad parts is a screwing process. Screws are subject to various manufacturing defaults (malformed thread, missing slot, remaining burr, bad length, ...). Let us consider a basic screwing process where one screw is inserted in a subassembly. We will neglect the value of the sub-assembly. Here are the values for all the parameters:

- $1 \%$ of the screws are malformed $(k=0.01)$.

- We neglect the sub-assembly value $\left(V_{s}=0\right)$.

- 1000 screws cost $10 €\left(V_{p}=0.001\right)$.

- The screwing machine can screw 1000 screws per hour $\left(T_{c}=3.6\right)$

- The feeder jams during $10 \mathrm{~s}\left(T_{j f}=10\right)$, and the screwing machine jams during $60 \mathrm{~s}\left(T_{j p}=60\right)$.

- The hourly costs are $30 € \mathrm{~h}$ for the screwing machine $\left(C_{\text {line }}=0.083\right)$ and $36 € \mathrm{~h}$ for an operator $\left(C_{o p}=0.01\right)$.

With these figures, the cost for assembling one screw is given by:

$$
C_{0}=0.03 ; C_{1}=0.043 ; C_{2}=0.0319 ; C_{3}=0.0411 ; C_{4}=0.0301
$$

In conclusion, if the screws jam in the screwing machine, it is not worth to sort the screws before feeding $\left(C_{1}>C_{3}\right)$, but we would accept to pay $20 €$ instead of $10 €$ for 1000 screws without any scrap.

Example 2 : plastic caps assembly. Plastic caps are glued on carton packages for juices and milk. They are made of 2 parts: the neck and the cap. The cap is inserted on the neck by press-fitting. Then, this sub-assembly is glued on the juice brick. Sometimes, the assembly is detected as bad because either the cap or the neck has an injection failure. The question is : Is it worth sorting the parts before assembly?

Here, we have the following parameters : $k=0.001, T c=0.2, V_{p}=$ $0.001, V_{s}=0.002, C_{\text {line }}=30$ If the line can sort the parts first, then $C_{\text {line }}=$ 35 We obtain then the costs:

$$
C_{0}=0.0022 ; C_{1}=0.0025 ; C_{2}=0.0024 ; C_{4}=0.0022
$$


$C_{3}$ is not evaluated because the press-fit will not stop if the parts are defective. $C_{2}$ is given for information, even if all bad parts would not jam in the feeder. The answer to our question is that it is not worth sorting the bad parts before feeding, since the cycle-time is small and the part value very low.

Example 3 : closing an aluminum housing with 6 screws. Let us consider a sensor packaged in an aluminum housing closed with 6 screws. $0.2 \%$ of the screws are defective (which is good) and will cause a jam and damage the housing. As we use 6 screws on each assembly, we use an new value of $k$ which is the probability to have at least one defective screw on an assembly $k_{6 \text { screws. }}$ If $k_{\text {screw }}=1 \%$, then

$$
k_{6 s c r e w s}=1-\left(1-k_{\text {screw }}\right)^{6}=1.2 \%
$$

If a jam occurs due to a malformed screw, the sub-assembly has to be reworked, with an average cost of $3.5 €$, including operator time and spare parts. The other parameters are the same as in example 1, and the model gives these results:

$$
C_{0}=0.050 ; C_{1}=0.086 ; C_{3}=0.112 ; C_{4}=0.100
$$

Here, we can evaluate the catastrophic consequences of using screws even with a small scrap in these conditions. $0.2 \%$ scrap that jam in assembly will double the assembly cost $\left(C_{3} / C_{0}=2.25\right)$ and cause $10 \%$ down time. It is far better to inspect and sort the screws before feeding them $\left(C_{1}\right)$, even if the bad screws do not jam the screwing-machine $\left(C_{4}\right)$.

\section{DISCUSSION AND CONCLUSION}

As the examples show, a small scrap in the parts can generate very large costs mainly due to the time loss during jams (example 1) and the loss of valuable good parts in bad assemblies (example 3 ). The model presented here allows a fast estimation of the costs.

This model should also be be useful to design new production lines and choose the inspection policy which minimizes the costs and down-time. It also provides a technique to evaluate the amount that is worth being spent for parts with a lower scraps.

This model is quite simple and does not include the following aspects:

- The model takes into account the hourly cost of operators, but the cost is biased, firstly because you must have an integer number of operators, secondly because it strongly depends on the operator policy: either he stands in front of the machine and can quickly fix a jam when it occurs, or he can perform other production tasks (filling the feeders for instance) and cause a delay in fixing the jam. However, there is no other solution 
than evaluating every assembly system depending on the tasks affected to its operators.

- The loss of productivity is evaluated for each assembly process of the line. This is useful to identify the bottleneck, but the overall productivity must be evaluated using analytical techniques (Buzacott and Shanthikumar, 1993; Hongler, 1994; Medhi, 2003) or discrete-events simulation (Heinzelmann, 1995)

\section{ACKNOWLEDGMENTS}

This work has been partially supported by the EUPASS project (funded by the European Commission), whose aim is to design and evaluate the performances of reconfigurable precision assembly systems.

\section{REFERENCES}

1. Buzacott, J.-A. and Shanthikumar, J.-G. (1993). Stochastic models of manufacturing systems. Prentice Hall.

2. Dubois, D. and Forestier, J. (1981). Productivité et en-cours moyens d'un ensemble de deux machines séparées par une zone de stockage. R.A.I.R.O. Autom. Syst. Analysis and Control, 16:105-132.

3. Gershwin, S. B. (1994). Manufacturing Systems Engineering. PTR Prentice Hall.

4. Han, M.-S. and Park, D.-J. (2002). Performance analysis and optimization of cyclic production lines. IIE Transactions, 34:411-422.

5. Heinzelmann, E. (1995). Simas ii - ein simulationswerkzeug für kmu. Technische Rundschau Transfer, 3:24-29.

6. Hong, C., Milor, L., Choi, M., and Lin, T. (2005). Study of area scaling effect on integrated circuit reliability based on yield models. Microelectronics reliability, 45(9-11):1305-1310.

7. Hongler, M. O. (1994). Chaotic and Stochastic Behaviour in Automatic Production Lines. Springer Verlag.

8. Jacot, J. (2004). Industrialisation, Notes de cours. EPFL.

9. Medhi, J. (2003). Stochastic Models in Queuing Theory. Academic Press.

10. Oulevey, M., Roduit, P., Koelemeijer, S., and Jacot, J. (2003). A cost model for flexible high speed assembly lines (erace project).

11. Terracol, C. and David, R. (1987). Performance d'une ligne composée de machines et de stocks intermédiaire. $A P I I, 21: 239$. 